\title{
LA EVOLUCIÓN DE LAS CIUDADES INTERMEDIAS EN LA ARGENTINA
}

\section{A EVOLUÇÃO DAS CIDADES INTERMÉDIAS NA ARGENTINA}

\author{
Fernando Ariel Manzano \\ Universidad Provincial de Ezeiza (UPE), Ezeiza, Buenos Aires, Argentina, fernandoarielmanzano@hotmail.com \\ Guillermo Angel Velazquez \\ Universidad Nacional del Centro de la Provincia de Buenos Aires (UNICEN), Tandil, Buenos Aires, Argentina, \\ gvelaz@fch.unicen.edu.ar
}

\section{RESUMEN}

Si bien no nos referimos a un fenómeno reciente, el proceso de urbanización transformó la organización de las ciudades y ayudó a reinventar la vida urbana en el mundo constantemente. En la actualidad, se aprecian cambios con respecto al típico patrón de dinámica demográfica del pasado, donde predominaba la migración del campo hacia las ciudades capitales. El avance de los medios de transporte y de las interconexiones virtuales, presenta un movimiento poblacional que no privilegia a las grandes urbes, y que genera un crecimiento de ciudades medianas, cuya particularidad radica en el estrecho vínculo de éstas con el mundo rural, no sólo desde lo productivo (neorruralidad). El objetivo de este trabajo es destacar la importancia de las ciudades intermedias en Argentina, muchas veces "invisibles" para todos, y que van cobrando dinamismo y fuerza, transformándose en centros de intercambio económico, fundamental para el desarrollo de algunas áreas rurales y urbanas menores.

Palabras claves: Migración; Urbanización; Ciudades intermedias; Neorruralidad.

\section{RESUMO}

Enquanto nós não estamos falando de um fenômeno recente, o processo de urbanização transformou a organização das cidades e ajudou a reinventar da vida urbana no mundo constantemente. Hoje, as mudanças são evidentes no que se refere ao padrão típico da dinâmica demográfica do passado, onde dominou a migração do campo para as cidades. A evolução dos meios de transporte e o virtual das interconexões, apresenta um movimento de população que não é favorável para as grandes cidades, e que gera um crescimento das cidades médias, cuja peculiaridade reside na estreita ligação com o mundo rural, não só do ponto de vista produtivo (neorruralidad). O objetivo deste trabalho é destacar a importância de cidades de médio porte na Argentina, muitas vezes "invisível" a todos, e que estão ganhando mais força e vigor, tornando-se centros de intercâmbio econômico, essencial para o desenvolvimento de algumas áreas rurais e urbanas as crianças.

Palavras-chave: Migração; Urbanização; Cidades Intermédias; Neorruralidad.

Artigo recebido para publicação em setembro de 2015

Artigo aceito para publicação em outubro de 2015

\section{INTRODUCCIÓN}

El usuario de la ciudad es un ser móvil a diferentes escalas. En efecto, el habitante camina pero además utiliza diferentes medios de transporte para poder vivir dentro de la ciudad contemporánea. Es necesario que los trabajos de investigación no sólo hagan referencia al barrio -por ejemplo el estudio de los sectores populares en los barrios de la periferia urbana-, sino también a los espacios públicos y a las zonas residenciales de los sectores medios o altos donde imperan la desigualdad y la subordinación como condiciones de relación social -en donde se vinculan actores de diferentes sectores sociales. 
Por tanto, los espacios de circulación son esenciales para entender el fenómeno urbano contemporáneo. Se debe avanzar en el análisis de la movilidad de los procesos urbanos, de manera de evitar correr el peligro de investigar de forma paralelala ciudad de la circulación y la ciudad de las residencias (VEGA CENTENO, 2004). Para ello vale la pena recordar la analogía de De Certeau, según la cual el caminar es a la ciudad lo que la enunciación a la lengua (1996).

Estudiar cómo se constituyen los espacios para desplazarse y cómo los habitantes de la ciudad diariamente realizan diferentes formas de desplazamiento, es uno de los grandes temas en donde las ciencias sociales debe avanzar para poder interpretar de mejor manera el proceso de urbanización -fenómeno que transformó la organización de las ciudades y ayudó a reinventar la vida urbana en el mundo-.

La formación de diferentes subcentros como polos de desarrollo residencial, se genera gracias al proceso de urbanización que experimenta la ciudad, donde los medios de transporte y las interconexiones virtuales, fueron fundamentales para comprender estas nuevas formas de crecimiento urbano. De esta manera es posible habitar lugares alejados del centro de trabajo y de la densidad de la vida que transcurre en las grandes ciudades.

Es necesario poder explicar las lógicas metropolitanas dispersas, por ejemplo las aglomeraciones veraniegas en los alrededores de las playas -aglomeraciones de menor escala pero que dependen de las dinámicas metropolitanas para su crecimiento-. Este fenómeno se aproxima a lo que algunos autores, como Bassand (2001), han definido como metropolización, donde las ciudades articulan tejidos nodales desligados del carácter compacto de una urbe. Hay, entonces, que prestar mucha atención a este fenómeno, que a primera vista puede confundirse con una lectura de procesos de ciudades intermedias. Se trata de fenómenos contemporáneos que se viven de manera muy particular, donde las condiciones de segregación y desigualdad social marcan las particularidades de las dinámicas urbanas.

\section{Algunas consideraciones metodológicas acerca de las ciudades intermedias}


Cuando hablamos de "ciudad" tendemos a referirnos en forma casi exclusiva a las grandes ciudades. De una dinámica demográfica en la que predominaba la migración del campo hacia las ciudades capitales, hemos pasado en pocas décadas a un movimiento poblacional que no privilegia sólo a las grandes urbes, contribuyendo al crecimiento de ciudades medianas o pequeñas, cuya particularidad radica en el estrecho vínculo de éstas con el mundo rural (neorruralidad). Las grandes ciudades hace tiempo que perdieron la capacidad de absorción de esta población que migra en busca de mayores oportunidades de educación y de trabajo ${ }^{1}$ (LLONA et al, 2004).

La delimitación de las ciudades con rígidos criterios de cantidad, resulta totalmente infructuosa. Una ciudad se considera intermedia no sólo con arreglo a tallas demográficas y dimensiones determinadas sino, sobre todo, con relación a las funciones que desarrolla: el papel de mediación en los flujos (bienes, información, innovación, administración, etc). entre los territorios rurales y urbanos de su área de influencia y los otros centros o áreas, más o menos alejadas. Funciones de intermediación entre los espacios locales/territoriales y los espacios regionales/nacionales e, incluso, globales (BELLET \& LLOP, 2004).

La dinámica de estas ciudades se mide por su capacidad para establecer una red de relaciones con los demás núcleos urbanos y entre los núcleos urbanos y el campo.

Cada ciudad existe en lo que denominamos un sistema de "ramilletes urbanos". En esta lógica, de corte más bien económico, la ciudad no puede ser entendida sin el campo y sin las otras ciudades con las que se complementa en sus funciones

En estos sistemas o redes, las ciudades intermedias y pequeñas, muchas veces "invisibles" para todos, van cobrando dinamismo y fuerza, se transforman en centros de intercambio económico -fundamental para el desarrollo de algunas áreas rurales y urbanas menores-, y de interacción social y cultural, en

\footnotetext{
${ }^{1}$ Los estudios de caso realizados demuestran que los jóvenes rurales han empezado a migrar a ciudades intermedias no sólo en bu sca de empleo sino también de la oferta de educación superior que allí se encuentra.
} 
donde se modifica significativamente la relación tradicional entre lo rural y lo urbano. Finalmente, son también espacios que albergan algunos niveles de la administración del gobierno a nivel local.

Dadas las características específicas de las ciudades intermedias, la incidencia de la pobreza, el empleo informal y de baja calidad, resulta más alta, producto de la mayor inversión pública concentrada en las metrópolis.

Las ciudades intermedias, por su propia escala, tienen la posibilidad de llevar a cabo proyectos de desarrollo territorial más sostenibles y de mejorar su función de aglomeración de servicios e infraestructura para las zonas rurales. En este sentido, las ciudades intermedias ofrecen una serie de nuevas oportunidades ya que funcionan como centros privilegiados para la provisión de servicios para el campo.

La modernización agropecuaria también ha significado un impacto en las ciudades intermedias, debido a que las migraciones del campo no se dirigen a las grandes ciudades y a la metrópoli necesariamente, sino que tienden a relocalizarse en pequeños poblados o ciudades medianas, sin romper el vínculo con las actividades agropecuarias -la movilidad poblacional interna entre centros urbanos menores también se ha incrementado-, (LLONA et al, 2004).

Las grandes urbes ya no logran insertar a los migrantes a su dinámica, ni asegurar puestos de trabajo estables. Debido a esto, los jóvenes migrantes que salieron a estudiar -mejor preparados- regresan a su lugar de origen en busca de oportunidades.

Resulta curioso que el concepto de ciudad intermedia, que sólo podemos definir vagamente, sea una realidad prácticamente universal: donde existe una mínima armadura urbana, hay ciudades intermedias. Estas suponen realidades diversas, presentes en todos los contextos, pero considerablemente menos visibles que las que configuran las grandes aglomeraciones urbanas (BELLET \& LLOP, 2004). 
Un trabajo realizado por el programa CIMES (BELLET \& LLOP, 2002, p.38-39), titulado "Ciudades intermedias y urbanización mundial", nos ayuda a definir el rol que una ciudad intermedia juega en su territorio, en una escala local/regional. En la investigación se destacan las siguientes características:

- Centros servidores de bienes y servicios más o menos especializados para la población del mismo municipio y de otros municipios más o menos cercanos sobre los que ejerce cierta influencia.

- Sistemas más equilibrados y sostenibles (por razones de escala) que ejercen relaciones más equilibradas con su territorio.

- Centros más fácilmente gobernables y que permiten en principio mayor participación ciudadana en el gobierno y gestión de la ciudad.

- Asentamientos que ayudan al ciudadano a identificarse más con su ciudad.

- Disminución de los problemas medioambientales en relación con las megaciudades.

- Bajos niveles de conflictividad social y menores costos sociales.

- Menor diversidad social y cultural, estimulan efectos de endogamia social.

- Baja competitividad económica frente a la metrópoli que tiende a concentrar las funciones superiores del sistema.

Teniendo en cuenta los procesos de mundialización de la economía, se destaca que la polarización generada tiende a penalizar a las ciudades medias y aglomeraciones urbanas que no están bien situadas en la red. Pero a su vez dejan una oportunidad a centros medianos y pequeños para resituarse en la red global. Con respecto a los principales factores que condicionan el rol de las ciudades intermedias en la red global, el programa CIMES destaca:

- La posición y posibilidades de conexión a las grandes redes y flujos.

- El grado de cohesión social, cooperación y voluntad de los principales agentes sociales de la ciudad.

- La atención que se brinde a la educación, preparación y calificación de sus ciudadanos.

- La capacidad creativa del medio y de adopción de innovaciones. 
- El nivel de relaciones de complementariedad y cooperación con otros nodos, con otros puntos, con otras ciudades -esta cooperación es básica frente a la concentración urbana mundial-.

- - La eficacia de una planificación física o urbanística en relación con el tamaño de las ciudades intermedias -su tamaño y dimensión urbana presentan ventajas con respecto a la gran ciudad-.

- -El avance de buenas políticas de movilidad urbana (buenos servicios y red de transporte público, políticas de peatonalización, regulación de tránsito rodado, descentralización de servicios y equipamiento de barrios, buen diseño de espacios libres y recorridos urbanos, etc.).

\section{El desarrollo de las ciudades intermedias en el mundo y la región.}

De acuerdo con los datos de Naciones Unidas (2002), entre el año 1950 y el 2000 la población mundial pasó de 2,52 a 6,06 billones de personas, un aumento del 140,5\% durante la segunda mitad del siglo XX. El porcentaje de población urbana en este intervalo de tiempo creció de 29,8\% a 47,2\%, es decir un crecimiento de $281,3 \%$ (de 0,75 a 2,86 billones de personas), mientras que la población rural creció sólo 80,2\% (de 1,77 a 3,19 billones de personas).

Con respecto a la región de América Latina y El Caribe, el porcentaje de población urbana era más alta que la mundial (para el año el año 1950 era de 41,9\% de la población total), la brecha entre la población urbana de América Latina y El Caribe y la mundial se amplió hasta 1980, en donde la primera era $64,4 \%$ superior $-65,1 \%$ y $39,6 \%$ eran la poblaciones urbanas, respectivamente-, a partir de ese entonces la diferencia comenzó a achicharse. Para el año 2000 el porcentaje de población urbana de América Latina y El Caribe era 75,4\%, un crecimiento relativo entre 1950 y 2000 de 458,6\%, pasando de 70 a 391 millones de personas -muy superior al nivel de urbanización que se dio en el mundo en este periodo-, mientras que la población rural creció sólo 30,9\%, de 97 a 127 millones de personas.

En 1950 sólo había una ciudad que superaba los 10 millones de habitantes: New York. Para 1975 las ciudades de esta talla demográfica eran 5 en el mundo, de las cuales 2 estaban en América Latina -México D.F. y San Pablo-, mientras que para el 2001 había 17 ciudades con más de 10 millones de 
habitantes, de las cuales 3 pertenecían a América Latina -San Pablo, Rio de Janeiro y Buenos Aires-, (Naciones Unidas, 2002).

Como se observa en la Figura 1 en 1980 la mayoría de la población urbana mundial (54,2\%), habitaba en ciudades menores a 500 mil habitantes y se estimaba que para el 2010 la proporción descendiese a $50,8 \%^{2}$.

Los aglomerados en los que residía el mayor porcentaje de población estaban comprendidos entre 1 y 5 millones; en 1980 residían el 22,1\% y en el 2010 alcanzarían 24,4\%. La cantidad de aglomerados de este tamaño, eran 212 en 1980 y aumentaron a 442 en 2010, lo que significó un crecimiento relativo del $108,5 \%$.

El tamaño de aglomerados urbanos más numeroso es entre 500 mil y 1 millón de habitantes; en 1980 eran 263 y se estimaba que para el año 2010 sean 495 -por tanto para el año 2010 continúan siendo los más numerosos, con una diferencia apenas superior a los aglomerados de tamaño de entre 1 y 5 millones.

Entre 1980 y 2010 las ciudades que más crecieron fueron las de más de 10 millones de habitantes, que pasaron de ser sólo 5 a un total de 20 durante estos 30 años, mientras que el porcentaje de población mundial que las habita se duplicó entre 1980 y 2010.

Por último la población que reside en ciudades entre 5 y 10 millones de habitantes disminuyó su participación de $9,0 \%$ en 1980 a $6,2 \%$ en el 2010, aunque la cantidad de aglomerados creció de 265 a 442, durante estos 30 años.

\footnotetext{
${ }^{2}$ El crecimiento de las grandes aglomeraciones urbanas y los procesos de concentración han resultado más moderados de lo que se preveía pocos años antes. Véase, por ejemplo, las previsiones que la ONU realizaba en 1996 (Naciones Unidas, 1997), cuando se apuntaba que, en el año 2000, en las ciudades de más de 10 millones residirían el 9,6\% de la población (7,9\% en la revisión del 2001).
} 


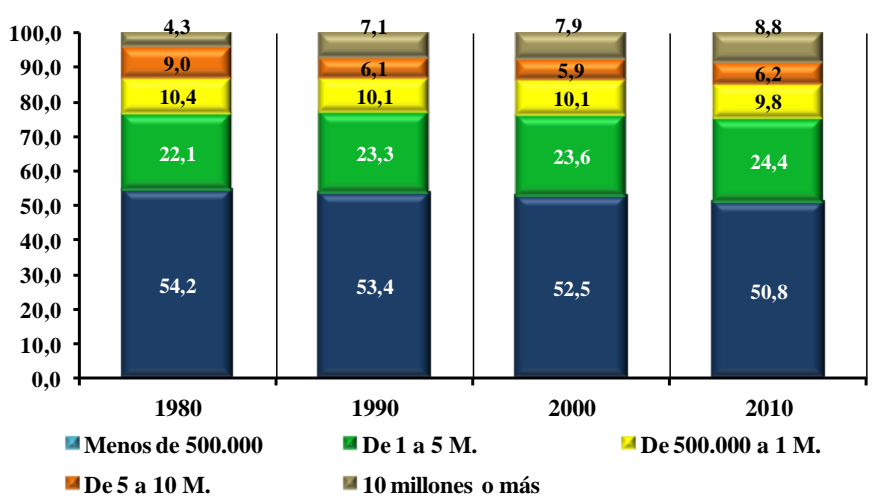

Figura 1. Distribución de población urbana según la dimensión del núcleo. Total Mundial. Años: 1980, 1990,2000 y 2010. En porcentaje. Fuente: Elaboración personal sobre la base de Naciones Unidas (2002).

La población que vive en ciudades de América Latina ha crecido a la vez que la tasa de urbanización se ha reducido casi a la mitad. Esto último se explica por la reducción de la tasa de migración ruralurbana -que ha dejado de ser el tema más significativo para explicar la evolución demográfica urbanay por la disminución del crecimiento vegetativo o interno en las ciudades.

En 1980 la mayoría de la población urbana de América Latina habitaba en zonas menores a 500 mil habitantes (se alojaba el 53,5\%), con una tendencia descendente, mayor a la observada a escala mundial. Así para el año 2010 la población de la región que habita estos espacios disminuyó a 43,4\%.

En América Latina los aglomerados en los que reside mayor porcentaje de población están comprendidos entre1 y 5 millones. En 1980 residía el 18,0\% y en el año 2010 alcanzaría el 25,9\%. La cantidad de aglomerados de este tamaño eran 22 en 1980 y aumentaron a 61 en 2010 -el crecimiento fue $177,3 \%$-. Tanto a nivel de crecimiento de población, como en la cantidad de aglomerados, el aumento es significativamente superior al total del mundo.

También la región coincide con la situación mundial con respecto a la escala de aglomerados urbanos más numerosos (entre 500 mil y 1 millón de habitantes). En 1980 eran 27 y en el 2010 son 62 -crecimiento relativo de 129,6\%-. Pero a diferencia de la situación a nivel total del mundo, el porcentaje de personas que residen en este tamaño de ciudades presenta un leve ascenso; en el año 1980 residía el 8,2\% y en el año 2010 aumentó al 9,5\%.Ver Figura 2. 
Con respecto a las ciudades de más de 10 millones de habitantes, entre 1980 y 2010 el porcentaje de población de la región que las habita pasó de 11,7\% en 1980 a 14,6\%. La cantidad de aglomerados de este tamaño en la región, también creció a menor ritmo que el mundial, pasando de 2 a 4 durante estos 30 años.

$\mathrm{Al}$ igual que en el mundo, la población urbana de la región que reside en ciudades entre 5 y 10 millones de habitantes disminuyó su participación de $8,0 \%$ en 1980 a $6,1 \%$ en el 2010,y la cantidad de aglomerados creció de 2 a 3, durante estos 30 años.

Los aglomerados que más crecieron en cantidad y en participación relativa en América Latina fueron los de 1 a 5 millones de habitantes, mientras que en el mundo fueron los de 10 millones o más.

En el año 2010, 40,9\% de la población mundial y 56,6\% de la población de América Latina vive en ciudades mayores a 500 mil habitantes. En este año4 de las 20 ciudades del mundo con más de 10 millones de habitantes se encuentran en América Latina (San Paulo, Ciudad de México, Buenos Aires y Rio de Janeiro).

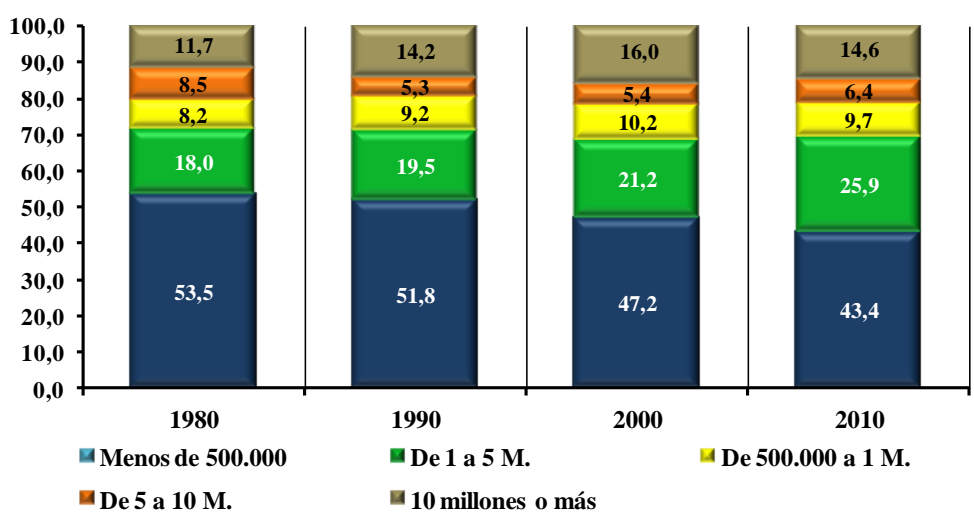

Figura 2. Distribución de población urbana según la dimensión del núcleo. América Latina. Años: 1980, 1990,2000 y 2010. En porcentaje. Fuente: Elaboración personal sobre la base de Naciones Unidas (2002).

\section{Los cambios en el sistema urbano Argentino.}


Durante los siglos XVI y XVII el noroeste argentino (NOA) era la región más dinámica y densamente poblada ya que reunía a más de la mitad de la población.

Antes de su surgimiento como Estado Nacional y del comienzo de la etapa agroexportadora, Argentina presentaba un proceso de desigualdad en la distribución de la población y las actividades económicas.

En el siglo XVIII, la apertura "legal" del puerto de Buenos Aires al tráfico de ultramar y el particular proceso de apropiación de la tierra en muy pocas manos, fueron claves en la explicación de la concentración de la población, de las inversiones y de la infraestructura en Buenos Aires y la región pampeana, así como del precoz proceso de urbanización de la Argentina.

Ya en los primeros censos nacionales (años 1869 y 1895) fue notorio el predominio de Buenos Aires en el sistema urbano argentino.

Resulta importante destacar que Argentina hasta aproximadamente el año 1937 cumple el rol de proveedor de alimentos y materias primas en el sistema de división internacional de la producción. Las inversiones de capital extranjero recibidas se volcaron a aprovecharlas "ventajas comparativas". La red ferroviaria argentina instalada fue diseñada para cumplir esta especialización, su eje era el puerto de Buenos Aires y el trazado en forma de "abanico", lo que implicaba una virtual barrera al comercio entre las regiones, concentrando la actividad económica en la región pampeana. De la mano del avance del modelo agroexportador ingresó una fuerte corriente migratoria ultramarina, cuya expectativa de acceder a la propiedad de la tierra, en la mayoría de los casos, no podía ser satisfecha, pues la tierra ya tenía dueño. Por tanto, los inmigrantes europeos (más de dos millones) terminaron radicándose principalmente en las grandes ciudades, generando un incremento notable del crecimiento urbano y la urbanización.

Con la crisis mundial de 1929 comienza a erosionarse el modelo agroexportador. Las condiciones internacionales generadas a partir del aislamiento provocado por los conflictos mundiales, condujeron a la instalación de un nuevo modelo productivo que trató de aprovechar las potencialidades del mercado 
interno sobre la base de un desarrollo industrial substitutivo de importaciones y con alta utilización de mano de obra.

Hubo una marcada concentración de inversiones y establecimientos industriales en la Región Metropolitana de Buenos Aires. A esto debemos sumarle la política del Estado en lo que respecta a créditos habitacionales que, en su mayoría, se dirigieron a apoyar la construcción de viviendas en el Gran Buenos Aires.

El nuevo golpe de estado en 1966 promovió la liquidación de economías regionales "ineficientes" como el Tucumán azucarero, o el Chaco algodonero.

Durante la década del cincuenta y sesenta se incrementaron significativamente las migraciones internas, fundamentalmente desde el Noroeste y Nordeste hacia el Gran Buenos Aires y la Región Pampeana. Este proceso de concentración de población por migraciones llevó al sistema urbano argentino al máximo nivel de primacía (más de 10 a 1 entre 1960 y 1970), y a un notable incremento de las desigualdades regionales, ya que el desarrollo manufacturero del interior, con salarios más bajos, se restringió casi exclusivamente a la transformación de productos primarios.

En el año 1950 Buenos Aires ya era la octava ciudad más poblada del mundo (más de 5 millones de habitantes) y tanto en 1955 como en 1960, se encontraba en el sexto lugar (con una población de 5,8y 6,7 millones, respectivamente).

A modo de comparación las cuatro ciudades de mayor cantidad de habitantes detrás de Buenos Aires en 1960, contaban con la siguiente población: Rosariotenía 671 mil habitantes -equivalente al 9,9\% de Buenos Aires-, en Córdoba residían 588 mil habitantes -el 8,7\% en relación a Buenos Aires-, Mendoza poseía 335 mil habitantes -el equivalente a 4,9\% de la población de Buenos Aires-, y en San Miguel de Tucumán eran 295 mil habitantes -el 4,4\% en relación a Buenos Aires- (Naciones Unidas, 2002). 
El diagnóstico de esta situación de primacía de Buenos Aires, trajo consigo la implementación de una serie de medidas de "desarrollo regional". La más destacable, durante la década del sesenta fue la creación del Consejo Nacional de Desarrollo (CONADE), que se proponía contribuir a un desarrollo regional más "equilibrado" a partir del establecimiento de "Regiones de Desarrollo", con sus respectivos "Polos" en el Noroeste (Salta), Nordeste (Corrientes-Resistencia), Centro (Córdoba), Cuyo (Mendoza), Pampeana (Rosario), Metropolitana (Buenos Aires), Comahue (Neuquén) y Patagonia (Comodoro Rivadavia $)^{3}$.

Durante la década del setenta surgió la propuesta de regímenes de promoción industrial en provincias extra-pampeanas, pero su implementación se concretó una década más tarde en dos provincias del Noroeste (Catamarca y La Rioja), dos de Cuyo (San Juan y San Luis) y un régimen especial en un territorio nacional patagónico (Tierra del Fuego).

Uno de los objetivos centrales de la dictadura de 1976 fue remover las bases del modelo de sustitución de importaciones. Este proceso afectó en mayor medida a las ciudades más grandes que contaban con mayor número de establecimientos industriales (fundamentalmente Buenos Aires, Rosario y Córdoba).

La restauración democrática a partir de 1983 generó importantes expectativas, pero el gobierno del Dr. Alfonsín termina enredado en un "Golpe de mercado", con niveles inéditos de pobreza, desocupación e hiperinflación.

El presidente Menem, fue su sucesor en 1989 y propuso asumir, sin eufemismos, el mundo unipolar de la "globalización". Con el objetivo de frenar la inflación puso en marcha el Plan de Convertibilidad, además de un conjunto de medidas ortodoxas, como privatizaciones, libertad al ingreso de capitales financieros especulativos, apreciación del tipo de cambio y disminución de aranceles a la importación. El resultado del modelo fue el aumento del desempleo a niveles históricos y un proceso de desmantelamiento de la trama productiva que afectó en mayor medida a las grandes ciudades.

\footnotetext{
${ }^{3}$ En el artículo 29 del estatuto del CONADE se establecen estas cabeceras regionales. (Argentina. Ministerio del Interior, 2002).
} 
En los últimos lapsos el ritmo de urbanización ha ido mermando. Como resulta lógico, a partir de cierto nivel de urbanización disminuye la tasa de crecimiento. El proceso de "eficientización económica", logró neutralizar el incremento de la primacía urbana de Buenos Aires.

Los cambios de tendencias más importantes en el sistema urbano argentino se verificaron a partir de los años setenta, cuando se produjo un aumento de la participación relativa de la población residente en las ciudades intermedias.

Como se observa en el Tabla 1, Argentina presenta un proceso de urbanización incipiente y acelerado en relación al contexto Latinoamericano. Durante el proceso agro exportador la tasa de crecimiento urbano alcanzó un valor de 53,6\%. A partir del nuevo régimen de acumulación basado en el proceso de industrialización y sustitución de importaciones, las tasas de urbanización llegaron a nuevos valores records -64,9\%о y 59,9\%o para los periodos 1947-1960 y 1960-1970, respectivamente-, así como también se dio comienzo a una tendencia creciente de despoblamiento de las zonas rurales.

\begin{tabular}{ccccccc}
\hline \multirow{2}{*}{ Censos } & \multicolumn{3}{c}{ Población } & \multicolumn{3}{c}{ Población (\%) } \\
\cline { 2 - 7 } & Total & Urbana & Rural & Total & Urbana & Rural \\
\hline 1869 & 1.830 .214 & 512,460 & 1.317 .754 & 100 & 28 & 72 \\
1895 & 4.044 .911 & 1.496 .617 & 2.548 .294 & 100 & 37 & 63 \\
1914 & 7.903 .662 & 4.188 .941 & 3.714 .721 & 100 & 53 & 47 \\
1947 & 15.893 .811 & 9.854 .163 & 6.039 .648 & 100 & 62 & 38 \\
1960 & 20.013 .793 & 14.409 .931 & 5.603 .862 & 100 & 72 & 28 \\
1970 & 23.364 .431 & 18.457 .900 & 4.906 .531 & 100 & 79 & 21 \\
1980 & 27.949 .480 & 23.198 .068 & 4.751 .412 & 100 & 83 & 17 \\
1991 & 32.615 .528 & 28.701 .665 & 3.913 .863 & 100 & SS & 12 \\
2001 & 36.260 .130 & 32.416 .556 & 3.843 .574 & 100 & 89 & 11 \\
2010 & 40.117 .096 & 36.065 .269 & 4.051 .827 & 100 & 90 & 10 \\
\hline
\end{tabular}

Tabla 1. Población Total, urbana y rural. En valores absolutos y porcentajes. Total de la Argentina. Años: 1869, 1895, 1914, 1947, 1960, 1970, 1980, 1991, 2001y 2010. Fuente: Censos Nacionales de 1869 a 2010. INDEC

Nota: Se considera "urbana" a aquella población que reside en localidades de 2.000 o más habitantes.

En 1950 la población urbana Argentina superaba el 62\%, mientras que a nivel mundial este porcentaje era de 29,8\% y en América Latina de 41,9\%. Para el año 2000 Argentina tenía un porcentaje de 
población urbana cercano al 90\%, mientras que en el mundo esta proporción era de 47,2 y en la región era de $75,4 \%$.

En la Figura 3, se observa que durante el último periodo intercensal, se produjo llamativamente un crecimiento de la población rural, dado que esta mantenía una continua reducción desde el año 1947. Consideramos que esto puede vincularse a los nuevos fenómenos de "neorruralidad": asentamientos por debajo de los 2.000 pero que poseen acceso a diversos servicios y cuentan con mayor nivel de tecnología; en general con población que proviene de zonas urbanas.

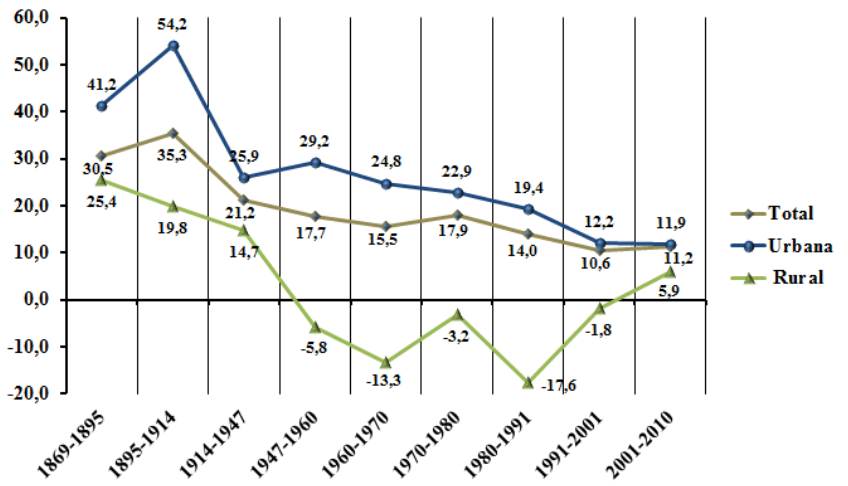

Figura 3. Tasa de crecimiento medio anual de la población total, urbana y rural (en miles), según censos de población. Total del país. Años 1869/2010. Fuente: Censos Nacionales de 1869 a 2010. INDEC.

\section{Evolución de las Aglomeraciones de tamaño intermedio (ATIs) en Argentina.}

El sistema urbano argentino en 1947 reflejaba la configuración espacial propia de la etapa agroexportadora, con predomino absoluto de la región pampeana y la participación minoritaria de las economías regionales tradicionales (NOA y Cuyo) y del NEA. Más de la mitad de las ciudades medias y grandes se localizaban en la región pampeana y ninguna en la Patagonia a pesar de los casi 70 años transcurridos desde la campaña militar contra los indígenas. La mitad de las provincias carecía de ciudades de este tipo.

El sistema urbano en 2010 muestra una situación bastante diferente, ya que las 67 ATIs se encuentran mucho más distribuidas entre las diferentes regiones argentinas, en relación a la situación 
a mitad del siglo pasado. Si bien el área pampeana sigue concentrando la mitad de las ciudades intermedias y grandes, todas las provincias argentinas cuentan con ciudades intermedias ${ }^{4}$.

El predominio de Buenos Aires en el sistema urbano argentino comenzó a revertirse en función del incremento de la participación relativa, cantidad y distribución regional de las ciudades de tipo intermedio (ATIs, comprendidas entre 50.000 y 1.000 .000 de habitantes, según el clásico trabajo de Vapñarsky y Gorojovsky, 1990) y el descenso de participación de la población dispersa y de aglomerados menores a 50.000 habitantes. Aunque desde1991, Córdoba y Rosario, hayan superado el millón de habitantes, la mayoría de los autores sigue considerándolas dentro del grupo de ciudades intermedias. Ver Tabla 2.

\begin{tabular}{ccccccc}
\hline Años & NOA & NEA & PATAG. & CUYO & PAMPA & TOTAL \\
\hline 1947 & 3 & 2 & - & 2 & 8 & 15 \\
1991 & 8 & 7 & 7 & 5 & 27 & 54 \\
2001 & 9 & 7 & 9 & 6 & 32 & 63 \\
2010 & 9 & 9 & 11 & 6 & 32 & 67 \\
\hline
\end{tabular}

Tabla 2. Distribución regional de las ciudades intermedias (ATIs). Fuente: elaboración personal sobre la base de Vapñarsky y Gorojovsky, 1990 y censos de población de Argentina (INDEC).

En el Tabla 3 se presenta la distribución de la población argentina en tres categorías de ciudades, ellas son:

I- Población dispersa y aglomerados menores a 50. 000 habitantes

II- Población en ATIs $^{5}$ (más de 50. 000 excepto GBA) ${ }^{6}$

III- GBA

La primera categoría correspondiente a la población dispersa y aglomerados con población menor a 50.000 habitantes presenta tendencia constante a la disminución de su participación. Entre el año 1947 y el 2010 lleva acumulada una caída de 24,0 puntos porcentuales.

\footnotetext{
${ }^{4}$ En 1991 Tierra del Fuego no contaba todavía con ninguna ciudad de 50.000 habitantes.

${ }^{5}$ Consideramos en este artículo como Aglomeraciones de Tipo Intermedio (ATIs) a las ciudades con menos de 1 millón de habitantes y más de 50.000 habitantes.

${ }^{6}$ El INDEC define al Gran Buenos Aires (GBA) como el área integrada por la Ciudad de Buenos Aires y los 24 partidos pertenecientes a los dos primeros grupos de partidos de la provincia de Buenos Aires que la rodean (INDEC, 2005).
} 
A su interior, disminuye la población rural aglomerada (núcleos de menos de 2.000 habitantes) y la de pueblos grandes (2.000 a 20.000), mientras que la población rural dispersa permanece más estable. Ello se debe al carácter históricamente despoblador de la actividad agropecuaria y a los efectos demográficos de la oferta de empleo urbano. Últimamente ejercen mayor influencia el efecto del transporte automotor y la telefonía celular, que permiten diferenciar el lugar de trabajo del de residencia, por lo que el término más correcto sería "mudanza", más que migración. En cambio la población de ciudades pequeñas (20.000 a 50.000 habitantes) aumenta a pesar de los "ascensos" de categoría que experimentaron algunas de estas ciudades durante este lapso. Como ser lo casos dentro de la región pampeana de Chivilcoy, Mercedes, Azul, el aglomerado de Villa Carlos Paz y General Pico, que ingresaron a la categoría II -ciudades de tamaño intermedio-, en el año 2001. Así como Tartagal en la región Noroeste y, en la región Nordeste, Clorinda y El Doradoen el 2010. Por último, en el caso de la región Patagonia, en el último periodo intercensal se sumaron Caleta Olivia y Ushuaia.

\begin{tabular}{|c|c|c|c|c|c|c|c|c|}
\hline $\begin{array}{c}\text { Categorías de } \\
\text { ciudades }\end{array}$ & Unidad & 1947 & 1960 & 1970 & 1980 & 1991 & 2001 & 2010 \\
\hline \multirow{3}{*}{$\begin{array}{c}\text { I-Pob. dispersa y } \\
\text { aglomerados } \\
\text { menores } 50.000 \\
\text { hab. }\end{array}$} & en miles & 9 & 9,150 & 9,100 & 10,060 & 10,679 & 12,075 & 11,805 \\
\hline & en $\%$ & 53,4 & 45,8 & 39 & 35,8 & 32,7 & 32,7 & 29,4 \\
\hline & TCMA(\%) & - & 1,3 & .0 .5 & 9,4 & 6 & 12,3 & 0,24 \\
\hline \multirow{3}{*}{$\begin{array}{c}\text { II-Pob. en ATIs } \\
\text { (más de } 50.000 \\
\text { excepto GBA) }\end{array}$} & en miles & 2,700 & 4,100 & 5,800 & 8,000 & 11, & 13 & 15 \\
\hline & en $\%$ & 16,0 & 20,5 & 24,8 & 28,6 & 33,7 & 36,3 & 36,7 \\
\hline & $\operatorname{TCMA}(\%)$ & - & 32,10 & 34,7 & 32,2 & 210 & 19,9 & 10,30 \\
\hline \multirow{3}{*}{ III-GBA } & en miles & 5,15 & 6,75 & 8,45 & 9,95 & 10,935 & 11,461 & 13,588 \\
\hline & en $\%$ & 30,6 & 33,8 & 36,2 & 35,6 & 33,5 & 31 & 33,9 \\
\hline & $\operatorname{TCMA}(\%)$ & - & 20,8 & 22,5 & 16,3 & 8,6 & 4,7 & 18,9 \\
\hline \multicolumn{2}{|c|}{ № de ATIs } & 15 & 23 & 31 & 41 & 54 & 63 & 67 \\
\hline \multirow{2}{*}{ Pob. Total del pais } & en miles & 15,894 & 20,014 & 23,364 & 27,949 & 32,616 & 36,26 & 40,117 \\
\hline & TCMA(\%) & - & 17,7 & 15,5 & 17,9 & 14,0 & 10,6 & 11,2 \\
\hline
\end{tabular}

Tabla 3. Distribución de la población argentina según categoría de ciudades (en miles de habitantes, en porcentaje respecto del total, tasa anual media de crecimiento) y evolución del número de Aglomeraciones de tamaño intermedio (ATIs). Fuente: elaboración personal sobre la base de Vapñarsky y Gorojovsky, 1990 y censos de población de Argentina (INDEC).

En relación con la segunda categoría (población en ciudades intermedias), resulta evidente el fuerte crecimiento -duplicando la tasa de crecimiento del total nacional-, con excepción del último periodo inter-censal, en donde la expansión estuvo por debajo de la media. 
Por último, la categoría III (correspondiente a la Región Metropolitana), muestra desde mediados de los años 1970, un descenso de su peso relativo -con excepción del último periodo intercensal en donde se produce una recuperación relativa-. Esta tendencia general podemos asociarla principalmente con las siguientes causas:

a) El modelo de exclusión que comenzó con la dictadura de 1976, de reestructuración productiva y concentración económica,

b) Des-economías de aglomeración, particularmente a lo que respecta a la provisión de servicios (agua, transporte público, eliminación de residuos, etc.) en el marco de la mercantilización agudizada tras su privatización y

c) Las cuestiones vinculadas con la calidad de vida (costo prohibitivo de vivienda, revalorización de lo “natural”, sensación de alienación e inseguridad, etc.).

En síntesis, durante los últimos 40 años el sistema urbano argentino pasó por una auténtica transformación. Actualmente la población de Argentina está dividida por tercios entre categorías de aglomeración, aunque las tendencias más regulares son: el descenso de la población dispersa, la participación de los aglomerados menores a 50.000 habitantes y el crecimiento de población residiendo en ATIs.

Durante el último período intercensal se aprecia un fenómeno distintivo, con cierta recuperación del peso relativo de la Región Metropolitana, probablemente asociado con la recuperación del aparato productivo durante la primera década del XXI.

A continuación analizaremos el comportamiento de las ciudades intermedias al interior de cada una de las regiones, excepto la Metropolitana de Buenos Aires que, naturalmente, carece de ciudades de esta categoría.

La región de Pampeana ha sido la de menor crecimiento. Durante el último periodo censal se destaca el crecimiento del Gran La Plata, así como el de ciudades cercanas a los 100 mil habitantes, como Tandil, 
Zarate, Lujan y Campana, que tuvieron un incremento por encima de la media de los aglomerados del Interior del GBA. En el caso de Córdoba, el crecimiento promedio de sus ciudades intermedias ha sido el más bajo de las 5 provincias que conforman la región pampeana, con la excepción del aglomerado Gran Carlos Paz. En la provincia de Entre Ríos los núcleos más chicos fueron los de mayor incremento. En la provincia de La Pampa el crecimiento se destaca en el caso del Gran Santa Rosa, mientras que en Santa Fe, en el aglomerado Reconquista -Avellaneda y Rafaela-. Ver Figura 4.

\begin{tabular}{|c|c|c|c|c|c|c|c|}
\hline \multirow[b]{2}{*}{ Regiones /Provincias/ Ciudades } & \multicolumn{4}{|c|}{ Población (en miles) } & \multicolumn{3}{|c|}{ Tasa de crecimiento medio anual (\%) } \\
\hline & 1947 & 1991 & 2001 & 2010 & $1947-1991$ & 1991-2001 & 2001-2010 \\
\hline Pampena & 2.462 & 5.867 & 6.767 & 7.374 & 19,7 & 14,3 & 9,5 \\
\hline Interior de Buenos Aires (promedio) & 923 & 2.181 & 2.508 & 2.787 & 19,5 & 14,0 & 11,7 \\
\hline Gran La Plata & 298 & 640 & 682 & 787 & 17,4 & 6,4 & 16,0 \\
\hline Mar del Plata & 135 & 520 & 542 & 593 & 30,6 & 4,1 & 10,1 \\
\hline Bahia Blanca & 121 & 255 & 272 & 291 & 16,9 & 6,5 & 7,6 \\
\hline San Nicolás de los Arroyos - B. Arroyo del Medio & 30 & 115 & 125 & 134 & 30,5 & 8,3 & 7,7 \\
\hline Tandil & 36 & 90 & 101 & 117 & 20,8 & 11,5 & 16,3 \\
\hline Zárate & 38 & 78 & 86 & 99 & 16,3 & 9,8 & 15,1 \\
\hline Luján & 21 & 65 & 78 & 97 & 25,7 & 18,2 & 24,6 \\
\hline Pergamino & 33 & 78 & 85 & 91 & 19,6 & 8,6 & 8,1 \\
\hline Olavarria & 27 & 73 & 83 & 90 & 22,6 & 12,8 & 8,7 \\
\hline Junin & 40 & 70 & 82 & 87 & 12,7 & 15,8 & 6,4 \\
\hline Campana & 18 & 67 & 78 & 87 & 29,9 & 15,2 & 12,0 \\
\hline Necochea - Quequén & 26 & 74 & 79 & 85 & 23,8 & 6,5 & 7,9 \\
\hline Punta Alta & 25 & 56 & 57 & 58 & 18,3 & 1,8 & 2,5 \\
\hline Chivilcoy & 24 & - & 53 & 58 & - & - & 10,3 \\
\hline Mercedes & 22 & - & 52 & 56 & - & - & 8,5 \\
\hline Azul & 29 & & 53 & 56 & & & 5,6 \\
\hline Córdoba & 537 & 1.476 & 1.736 & 1.860 & 23,0 & 16,2 & 7,7 \\
\hline Gran Córdoba & 416 & 1.195 & 1.368 & 1.455 & 24,0 & 13,5 & 6,8 \\
\hline Gran Rio Cuarto & 54 & 138 & 149 & 163 & 21,3 & 7,7 & 10,0 \\
\hline Villa Maria - Villa Nueva & 39 & 78 & 89 & 98 & 15,8 & 13,2 & 10,9 \\
\hline Villa Carlos Paz - San Antonio de Arredondo - Villa Rio Icho & & $t$ & 61 & 70 & - & - & 15,0 \\
\hline San Francisco - Frontera & 28 & 65 & 69 & 74 & 19,1 & 6,0 & 7,9 \\
\hline Entre Rios & 198 & 449 & 524 & 569 & 18,6 & 15,4 & 9,1 \\
\hline Gran Parana & 87 & 212 & 248 & 264 & 20,2 & 15,7 & 7,0 \\
\hline Concordia & 49 & 116 & 137 & 149 & 19,6 & 16,6 & 9,7 \\
\hline Gualeguaychú - Pueblo General Belgrano & 32 & 65 & 75 & 83 & 16,1 & 14,3 & 10,8 \\
\hline Concepción del Uruguay & 30 & 56 & 64 & 73 & 14,2 & 13,4 & 13,9 \\
\hline La Pampa & 25 & 81 & 154 & 171 & 26,7 & 64,3 & 11,8 \\
\hline Gran Santa Rosa & 19 & 81 & 102 & 114 & 33,0 & 23,1 & 12,8 \\
\hline General Pico & 6 & - & 52 & 57 & & -1 & 9,8 \\
\hline Santa Fe & 779 & 1.680 & 1.845 & 1.987 & 17,5 & $\mathbf{9 , 4}$ & 8,2 \\
\hline Gran Rosario & 543 & 1.096 & 1.159 & 1.236 & 16,0 & 5,6 & 7,2 \\
\hline Gran Santa Fe & 180 & 395 & 452 & 490 & 17,9 & 13,5 & 9,0 \\
\hline Reconquista - Avellaneda & 14 & 63 & 83 & 94 & 34,2 & 27,6 & 13,7 \\
\hline Rafaela & 26 & 67 & 82 & 92 & 21,5 & 20,2 & 12,3 \\
\hline Venado Tuerto & 16 & 59 & 69 & 75 & 29,7 & 15,7 & 9,9 \\
\hline
\end{tabular}

Figura 4. Ciudades intermedias en la región Pampeana. Años (1947-2001) población (en miles de habitantes) y tasa anual media de crecimiento (\%).Fuente: Argentina, 1949 y Argentina. INDEC, 1994, 2002 y 2012.

Con respecto al comportamiento de las ATIs en la región Cuyo, es el segundo menor -solo superior al de la región de Pampeana-. En la Figura 5, se presenta el comportamiento de cada una de las ciudades intermedias de la región, se destaca el crecimiento superior a la media en las ciudades de San Rafael, Villa Mercedes y del aglomerado Gran San Luis. 


\begin{tabular}{|c|c|c|c|c|c|c|c|}
\hline \multirow{2}{*}{ Regiones/Provincias/Ciudades } & \multicolumn{4}{|c|}{ Población (en miles) } & \multicolumn{3}{|c|}{ Tasa de crecimiento medio anual (\%o) } \\
\hline & 1947 & 1991 & 2001 & 2010 & $1947-1991$ & 1991-2001 & 2001-2010 \\
\hline Cuyo & \begin{tabular}{l|l}
453 \\
\end{tabular} & 1.481 & 1.711 & 1.899 & 26,9 & 14,4 & 11,6 \\
\hline San Rafael & 34 & 95 & 105 & 118 & 23,4 & 10,0 & 13,0 \\
\hline San Martin - La Colonia & 14 & 72 & 79 & 89 & 37,2 & 9,3 & 13,1 \\
\hline$\overline{\text { San Juan }}$ & 124 & 353 & 421 & 461 & 23,8 & 17,6 & 10,1 \\
\hline Gran San Luis & 28 & 110 & 162 & 182 & 31,1 & 38,7 & 13,2 \\
\hline Villa Mercedes & 28 & 77 & 97 & 111 & 23,0 & 23,1 & 15,4 \\
\hline
\end{tabular}

Figura 5. Ciudades intermedias en la región de Cuyo. Años (1947-2001) población (en miles de habitantes) y tasa anual media de crecimiento (\%o).Fuente: Argentina, 1949 y Argentina. INDEC, 1994, 2002 y 2012.

El Noroeste es la tercera región de mayor crecimiento, se destacan el aumento durante el último periodo censal de los aglomerados Gran San Fernando del Valle de Catamarca, Gran Salta, La Rioja y Tartagal. Ver Figura 6.

\begin{tabular}{|c|c|c|c|c|c|c|c|}
\hline \multirow{2}{*}{ Regiones /Provincias/Ciudades } & \multicolumn{4}{|c|}{ Población (en miles) } & \multicolumn{3}{|c|}{ Tasa de crecimiento medio anual (\%) } \\
\hline & 1947 & 1991 & 2001 & 2010 & $1947-1991$ & 1991-2001 & 2001-2010 \\
\hline Noroeste & 511 & 1.777 & 2.304 & 2.593 & 28,3 & 26,0 & 13,1 \\
\hline Catamarca & 38 & 133 & 171 & 195 & 28,5 & 25,1 & 14,6 \\
\hline Gran San Fernando del Valle de Catamarca & 38 & 133 & 171 & 195 & 28,5 & 25,1 & 14,6 \\
\hline Jujuy & 40 & 233 & 333 & 372 & 40,0 & 35,7 & 12,4 \\
\hline Gran San Salvador de Jujuy & 34 & 183 & 278 & 310 & 38,3 & 41,8 & 12,1 \\
\hline \begin{tabular}{|l|} 
San Pedro \\
\end{tabular} & 6 & 50 & 55 & 62 & 48,2 & 9,5 & 13,9 \\
\hline La Rioja & 27 & 104 & 144 & 179 & 30,6 & 32,5 & 24,1 \\
\hline La Rioja & 27 & 104 & 144 & 179 & 30,6 & 32,5 & 24,1 \\
\hline Salta & 93 & 421 & 592 & 692 & 34,3 & 34,1 & 17,3 \\
\hline Gran Salta & 77 & 370 & 469 & 551 & 35,7 & 23,7 & 17,9 \\
\hline San Ramón de la Nueva Orán (Est. Orán) & 7 & 51 & 67 & 76 & 45,1 & 27,3 & 14,1 \\
\hline Tartagal & 9 & - & 56 & 65 & - & 1 & 15,7 \\
\hline Santiago del Estero & 85 & 264 & 328 & 361 & 25,8 & 21,7 & 10,6 \\
\hline Santiago Del Estero - La Banda & 85 & 264 & 328 & 361 & 25,8 & 21,7 & 10,6 \\
\hline \begin{tabular}{|r|r|r|} 
& Tucumán \\
\end{tabular} & 228 & 622 & 736 & 794 & 22,8 & 16,8 & 8,5 \\
\hline Gran San Miguel de Tucuman & 228 & 622 & 736 & 794 & 22,8 & 16,8 & 8,5 \\
\hline
\end{tabular}

Figura 6. Ciudades intermedias en la región de Noroeste. Años (1947-2001) población (en miles de habitantes) y tasa anual media de crecimiento (\%o).Fuente: Argentina, 1949 y Argentina. INDEC, 1994, 2002 y 2012.

La segunda región donde mayor crecimiento han tenido las ATIs es el Nordeste, se destaca el crecimiento entre 2001 y 2010 de dos ciudades: Presidencia Roque Sáenz Peña y Oberá. Tal como se observa en el Figura 7, se han incorporado dos nuevas ciudades intermedias durante el último periodo inter-censal: Clorinda en Formosa y El Dorado en Misiones, acumulando un total de 9, al igual que la región Noroeste. 


\begin{tabular}{|c|c|c|c|c|c|c|c|}
\hline \multirow[b]{2}{*}{ Regiones /Provincias/ Ciudades } & \multicolumn{4}{|c|}{ Población (en miles) } & \multicolumn{3}{|c|}{ Tasa de crecimiento medio anual (\%) } \\
\hline & 1947 & 1991 & 2001 & 2010 & $1947-1991$ & $1991-2001$ & 2001-2010 \\
\hline Nordeste & 255 & 1.034 & 1.346 & 1.610 & 31,8 & 26,4 & 19,9 \\
\hline Corrientes & 86 & 315 & 382 & 418 & 29,5 & 19,3 & 10,0 \\
\hline Gran Corrientes & 65 & 258 & 316 & 346 & 31,3 & 20,3 & 10,2 \\
\hline Goya & 21 & 57 & 66 & 72 & 22,7 & 14,7 & 9,1 \\
\hline Chaco & 98 & 354 & 435 & 476 & 29,2 & 20,6 & 9,9 \\
\hline Gran Resistencia & 75 & 291 & 359 & 386 & 30,8 & 21,0 & 8,0 \\
\hline Presidencia Roque Sáenz Peña & 23 & 63 & 76 & 90 & 22,9 & 18,8 & 18,6 \\
\hline Formosa & 21 & 154 & 198 & 275 & 45,3 & 25,1 & 36,5 \\
\hline Formosa & 21 & 154 & 198 & 222 & 45,3 & 25,1 & 12,8 \\
\hline Clorinda & - & - & - & 53 & - & - & \\
\hline Misiones & 50 & 211 & 331 & 441 & 32,7 & 45,0 & 32,0 \\
\hline Gran Posadas & 45 & 211 & 280 & 319 & 35,1 & 28,3 & 14,7 \\
\hline Oberá & 5 & - & 51 & 65 & - & - & 26,4 \\
\hline El dorado & - & - & - & 57 & -1 & - & - \\
\hline
\end{tabular}

Figura 7. Ciudades intermedias en la región Nordeste. Años (1947-2001) población (en miles de habitantes) y tasa anual media de crecimiento (\%).Fuente: Argentina, 1949 y Argentina. INDEC, 1994, 2002 y 2012.

La región con mayor crecimiento de ATIs ha sido Patagonia -única región que tuvo crecimiento superior al del periodo intercensal anterior- destacándose la ciudad de Puerto Madryn. La región también ha sumado dos nuevas ciudades intermedias: Caleta Olivia en Santa Cruz y Ushuaia en Tierra del Fuego, llegando a un total de 11 ATIs, constituyendo así la segunda región con mayor cantidad -aunque muy por detrás del primer puesto perteneciente a la región pampeana con 32 ciudades intermedias-. Ver Figura 8.

\begin{tabular}{|c|c|c|c|c|c|c|c|}
\hline \multirow{2}{*}{ Regiones /Provincias/ Ciudades } & \multicolumn{4}{|c|}{ Población (en miles) } & \multicolumn{3}{|c|}{ Tasa de crecimiento medio anual (\%o) } \\
\hline & 1947 & 1991 & 2001 & 2010 & $1947-1991$ & 1991-2001 & 2001-2010 \\
\hline Patagonia & 88 & 709 & 928 & 1.230 & 47,4 & 26,9 & 31,3 \\
\hline Chubut & 39 & 202 & 282 & 354 & 37,4 & 33,4 & 25,4 \\
\hline Comodoro Rivadavia & 28 & 124 & 136 & 175 & 33,8 & 9,2 & 28,1 \\
\hline Trelew & 7 & 78 & 88 & 98 & 54,8 & 12,1 & 11,9 \\
\hline Puerto Madryn & 4 & & 58 & 81 & - & - & 37,5 \\
\hline Neuquén & 18 & 244 & 290 & 341 & 59,2 & 17,3 & 18,1 \\
\hline Neuquén - Plotier - Cipolletti & 18 & 244 & 290 & 341 & 59,2 & 17,3 & 18,1 \\
\hline $\begin{array}{r}\text { Río negro } \\
\end{array}$ & 25 & 198 & 224 & 264 & 47,0 & 12,3 & 18,3 \\
\hline San Carlos de Bariloche & 7 & 78 & 89 & 109 & 54,8 & 13,2 & 22,8 \\
\hline General Roca & 8 & 62 & 70 & 82 & 46,5 & 12,1 & 16,9 \\
\hline Viedma - Carmen de Patagones & 10 & 58 & 65 & 73 & 40,0 & 11,4 & 13,4 \\
\hline Santa Cruz & 6 & 65 & 79 & 148 & 54,2 & 19,5 & 69,4 \\
\hline Rio Gallegos & 6 & 65 & 79 & 96 & 54,2 & 19,5 & 21,4 \\
\hline Caleta Olivia & - & - & - & 52 & - & - & 4 \\
\hline Tierra del Fuego & 0 & 0 & 53 & 123 & - & & 93,6 \\
\hline Rio Grande & - & - & 53 & 66 & - & - & 25,2 \\
\hline Ushuaia & - & - & - & 57 & - & - & \\
\hline
\end{tabular}

Figura 8. Ciudades intermedias en la región Patagónica. Años (1947-2001) población (en miles de habitantes) y tasa anual media de crecimiento (\%).Fuente: Argentina, 1949 y Argentina. INDEC, 1994, 2002 y 2012.

La dinámica demográfica argentina tiene tendencias claras: disminución de la tasa de crecimiento vegetativo y migratorio, envejecimiento y creciente feminización de los flujos migratorios. Estas tendencias también podrían contribuir a explicar el mayor crecimiento relativo de las ATIs, ya que la 
disminución del crecimiento vegetativo implicó también cierta merma en las diferencias de fecundidad rural-urbana.

La combinación de des-economías de aglomeración, propensión a migrar por motivos no laborales y las tendencias estructurales de la población argentina, además de contribuir al mayor crecimiento relativo de las ATIs, da como resultado cierto proceso de redistribución interprovincial de población.

Este fenómeno se produce, sin embargo, a expensas de la población de los respectivos interiores provinciales, generando así mayor concentración intra-provincial; es decir se repite el mismo y criticado esquema de primacía urbana, pero esta vez, al interior de las provincias.

Con este nuevo sistema urbano la población de casi cualquier lugar de la Argentina tiene mayores posibilidades de obtener a menor distancia los bienes o servicios que pueda requerir. Además de la considerable ampliación de servicios sanitarios de alta y media complejidad en el territorio, por primera vez, todas las provincias argentinas poseen sus propias universidades nacionales ${ }^{7}$. En otros términos: la red de 67 ATIS y el incremento de la participación del Estado permitieron achicar considerablemente las distancias para acceder a servicios más especializados.

La existencia de 67ATIs puede abrir la posibilidad de beneficiar a una proporción de población mucho mayor que durante las etapas precedentes. El mayor crecimiento relativo de las ciudades intermedias en un contexto de mayor progresividad social como en el que se desarrolla en la Argentina desde el 2003 implicó mejoras en la calidad de vida de la población. Durante los noventa habían aparecido en las ciudades intermedias nuevos fenómenos como: la privatización de espacios públicos, la coexistencia de villas miseria y countries privados, la utilización de recursos públicos para los sectores de privilegio o el incremento de la inseguridad urbana, que antes eran fenómenos típicos de grandes ciudades (Velázquez, 1999).La situación desde ese entonces está mejorando. Los cambios operados a partir del 2003 están revirtiendo de alguna medida estos procesos, de forma tal que las ciudades intermedias

\footnotetext{
${ }^{7}$ La única provincia que carecía de su propia Universidad Nacional era Tierra del Fuego. Esta situación fue subsanada a partir del 2010.
} 
podrían volver a potenciar sus ventajas, fundamentalmente en lo que respecta a las posibilidades que pueden brindar para incrementar el bienestar de la población.

\section{CONCLUSIONES}

Si bien no nos referimos a un fenómeno reciente, el proceso de urbanización transformó la organización de las ciudades y ayudó a reinventar la vida urbana en el mundo constantemente.

En la actualidad, el avance de los medios de transporte y de las interconexiones virtuales, genera nuevas formas de crecimiento urbano y se experimenta el surgimiento de diferentes subcentros (polos) de desarrollo residencial.

Se aprecian cambios con respecto al típico patrón de dinámica demográfica del pasado, donde predominaba la migración del campo hacia las ciudades capitales. En las últimas décadas, se destaca un movimiento poblacional que no privilegia a las grandes urbes, y que genera un crecimiento de ciudades medianas, cuya particularidad radica en el estrecho vínculo de éstas con el mundo rural, no sólo desde lo productivo (neorruralidad).

Debemos prestar atención en no categorizar las ciudades con rígidos criterios de cantidad, ya que puede resultar totalmente infructuosa en muchos casos. Para las ciudades intermedias no sólo se debe tener en cuenta sus tallas demográficas, sobre todo, las funciones que desarrollan -su capacidad para establecer una red de relaciones entre los demás núcleos urbanos y entre los núcleos urbanos y el mundo rural-.

El propósito de este trabajo ha sido destacar la importancia de las ciudades intermedias, muchas veces "invisibles" para todos, y que van cobrando dinamismo y fuerza, transformándose en centros de intercambio económico, fundamental para el desarrollo de algunas áreas rurales y urbanas menores, de interacción social y cultural, en donde se modifica significativamente la relación tradicional entre lo rural y lo urbano. 
Las ciudades intermedias, por su propia escala, tienen la posibilidad de llevar a cabo proyectos de desarrollo territorial más sostenibles y de mejorar su función de aglomeración de servicios e infraestructura para las zonas rurales.

Resulta curioso que el concepto de ciudad intermedia, que sólo podemos definir vagamente, sea una realidad prácticamente universal: donde existe una mínima armadura urbana, hay ciudades intermedias.

Argentina se destaca en el contexto Latinoamericano por el acelerado proceso de urbanización.

En 1950 la población urbana Argentina era superior al 62\%, mientras que a nivel mundial este porcentaje era de 29,8\% y en América Latina de 41,9\%. Para el año 2000 Argentina tenía un porcentaje de población urbana cercano al 90\%, en el mundo esta proporción era de 47,2 y en la región de $75,4 \%$.

Durante las décadas del cincuenta y sesenta el sistema urbano argentino alcanza el máximo nivel de primacía (más de 10 a 1 entre 1960 y 1970), junto con un notable incremento de las desigualdades regionales.

La dictadura de 1976 tuvo como uno de sus objetivos centrales remover las bases del modelo de sustitución de importaciones. Este proceso afectó en mayor medida a las ciudades más grandes que contaban con mayor número de establecimientos industriales (fundamentalmente los casos de Gran Buenos Aires, Gran Rosario y Gran Córdoba). El resultado fue que el ritmo de urbanización comience a mermar, así como también la primacía urbana de Buenos Aires. La contra cara de esta dinámica, han sido el aumento de la participación relativa de la población residente en las ciudades intermedias y un creciente de despoblamiento de las zonas rurales. 
Considerando el periodo 1947-2010 la población dispersa y los aglomerados con población menor a 50.000 habitantes llevan acumulados una caída de 24,0 puntos porcentuales. La población en las ciudades intermedias se destaca por su fuerte crecimiento, ya que su participación relativa creció durante este periodo 20,7 puntos. Mientras que el Área Metropolitana sólo aumento su participación relativa en el total del país en 3,3 puntos.

A diferencia del pasado, en las últimas cuatro décadas la población de Argentina se encuentra dividida en aproximadamente tres tercios entre las categorías de aglomeración analizadas, aunque las tendencias que se destacan como más regulares son el descenso de la población dispersa, de aglomerados menores a 50.000 habitantes y el crecimiento de población residiendo en las ATIs.

Finalmente, si diferenciamos las ciudades intermedias según localización, el mayor crecimiento fue en la Patagonia. La región presenta en el año 2010 un total de 11 ATIs, mientras que en 1947 no tenía aún ninguna.

\section{REFERÊNCIAS}

ARGEnTINA. Ministerio del Interior. Secretaría de Provincias. La Regionalización en la Argentina Apuntes para su debate. Buenos Aires, 2002.

BASSAND, M.; KAUFFMAN, V.; JOYE, D. Enjeux de la sociologie urbaine. Presses Polytechniques et Universitaires Romandes (PPUR). Lausana, 2001.

BELLET, C. y LLOP, J. M. Las líneas de trabajo del programa UIA-CIMES: ciudades intermedias y urbanización mundial. En: Las nuevas funciones urbanas: gestión para la ciudad sostenible. CEPAL - SERIE Medio ambiente y desarrollo, 2002.

BELLET, C. y LLOP J. M. Miradas a otros espacios urbanos: Las ciudades Intermedias. Revista electrónica de geografía y ciencias sociales. Vol. VIII, No. 165, 15 de mayo de 2004. Disponible en internet: http://www.ub.edu/geocrit/sn/sn165.htm

DE CERTEAU, M. La invención de lo cotidiano. Vol.1: Artes de hacer. México: Universidad Iberoamericana, 1996.

INDEC ¿Qué es el Gran Buenos Aires?, 2005. Disponible en internet:

http://www.indec.gov.ar/nuevaweb/cuadros/1/folleto\%20gba.pdf

LLONA M; RAMÍREZ CORZO, D; ZOLEZZI, M. "Las ciudades intermedias: Su rol en el desarrollo del país". En Perú hoy. Las ciudades en el Perú. Desco, 2004. Disponible en internet:

http://biblioteca.clacso.edu.ar/ar/libros/peru/desco/peruhoy06.pdf

NACIONES UNIDAS. Urbanization prospects: The 1996 revision, United Nations, 1997. 
NACIONES UNIDAS. "World Urbanization Prospects The 2001 Revision Data Tables and Highlights". Population Division Department of Economic and Social Affairs United Nations Secretariat, ESA/P/WP.173 20 de marzo de 2002. Disponible en internet: http://www.un.org/esa/population/publications/wup2001/wup2001dh.pdf

VEGa Centeno, P. "De la barriada a la metropolización”. En: Perú hoy. Las ciudades en el Perú. Desco, 2004. Disponible en internet: http://biblioteca.clacso.edu.ar/ar/libros/peru/desco/peruhoy06.pdf

VAPÑARSKY, C. A. y GOROJOVSKY, N. El crecimiento urbano en la Argentina. Buenos Aires: Grupo Editor Latinoamericano-IIED, 1990.

VELÁZQUEZ, G. A y GARCÍA, M.C. Calidad de vida urbana. Aportes para su estudio en Latinoamérica. Tandil, CIG. 1999. 